-Nasopharyngeal Carcinoma Column.

\title{
Comment for the Chinese 2008 staging system for nasopharyngeal carcinoma
}

Ying Sun ${ }^{1,2}$ and Jun $\mathrm{Ma}^{1,2}$

1. State Key Laboratory of

Oncology in South China,

Guangzhou, Guangdong, 510060,

P. R. China

2. Department of Radiation Oncology, Cancer Center,

Sun Yat-sen University, Guangzhou 510060,

P. R. China

\author{
Correspondence to : Jun MA \\ Tel. : 86.20.87343469 \\ Fax. : 86.20.87343392 \\ Email:drjunma@hotmail.com
}

This paper was translated into English from its original publication in Chinese.

Translated by:Guangzhou Liheng and Wei Liu

The original Chinese version of this paper is published in:Ai Zheng (Chinese Journal of Cancer) 28(10);http://www.cjcsysu.cn/cn/ article.asp?id=16487)

Submitted: 2009-08-19

Revised : 2009-08-26

\begin{abstract}
[Abstract] The advantages of the Chinese 2008 staging system for nasopharyngeal carcinoma included as follows: (1)Application of MRI as the major staging means; discard of the subjective factors. (2) Adoption of some new independent predictor such as metastatic retropharyngeal lymph nodes and RTOG cervical levels. (3) Simplification of the T category. (4) Categorization of $\mathrm{N}$ and clinical substage can distinct the risk of distant metastasis and overall survival. All these changes adapted to the modern diagnosis and treatment pattern with a higher degree of practicality. Therefore, developing a prospective, multicenter clinical verification of the existing standards, thereby correcting itself, fulfill the developmental and scientific principles. It may improve as follows: (1)The definition of anatomical structure is restrictive such as nasal cavity and oropharynx, and the expression of masticator space is not intuitive. (2)Proof of evidence-based medicine for the size of lymph nodes included in the $\mathrm{N}$ category is insufficient. (3) The risk of local recurrence between the subgroups of $\mathrm{T}$ category was not significant. (4)Different definition of the index such as oropharynx and cervical level between the sixth edition UICC / AJCC staging system and the current system will hinder the international exchange of experience and information.
\end{abstract}

Key words: nasopharyngeal carcinoma, clinical stage

The objective of clinical staging of malignant tumor is to predict prognosis, guide treatment and evaluate the efficacy, and benefit comparison of data and exchange of experience among various centers. Due to particularity of nasopharyngeal carcinoma (NPC) in Chinese patients, Chinese scholars have devoted to develop the staging criteria suitable for Chinese patients. Since Tianjin staging was established in 1959, Chinese scholars have developed Shanghai staging in 1965, Changsha staging in 1979 and Fuzhou staging in 1992. The 92 Fuzhou staging is designed on the basis of CT diagnosis, two-dimensional conventional radiotherapy, simple radiotherapy, and so on. It played a crucial role in guiding treatment in 1990s. With the wide use of modern imaging techniques such as MRI, three-dimensional and intensity modulated radiotherapy technology as well as combined chemoradiotherapy, drawbacks gradually exposed in the 92 staging.

On 26 December 2008, Committee of Chinese Clinical Staging of Nasopharyngeal Carcinoma was established by Branch Association for Radiation Oncology Therapy of China Medical Association, Professional Committee for Radiation Oncology of Chinese Anti-Cancer Association and Professional Committee for Nasopharyngeal Carcinoma of Chinese Anti-Cancer Association. Revision of Clinical Staging of Nasopharyngeal 
Carcinoma was carried out through group discussion, and 2008 Chinese Staging of Nasopharyngeal Carcinoma (the 2008 staging) was established subsequently. ${ }^{1}$ The current article was to analyze the 2008 staging in terms of the staging evaluation measures, index screening and principles of staging, and to help clinicians understand the general background information of staging, facilitate its application and provide references for improving the staging.

\section{Improvement of the 2008 staging}

MRI become principal measure in clinical staging. In the development of TNM staging system, the reliability of staging, that is, certainty factors ( $C$ factors), has been changing along with the progresses of examination methods. C factors, the certainty factors of diagnosis in staging, reflects the authenticity of a diagnostic method. C1 refers to clinical examination and conventional $X$-ray examination (the Changsha staging); $C 2$ refers to the CT imaging examination (the 92 Fuzhou staging) or MRI (the 2008 staging), and so on; C3 refers to exploratory operation, including biopsy and cytology; C4 refers to pathologic examination; C5 refers to autopsy. NPC is not suitable to be treated by surgical operation because of the deep anatomical location of the nasopharynx. As a result, evidences from C3-C5 levels are difficult to obtain. In the meantime, it is difficult to recognize the invasion of the parapharyngeal space, skull base and intracranial cavity by clinical examinations, therefore, imaging examinations such as $\mathrm{CT}$ and $\mathrm{MRI}$ are required. According to several reports on comparative study of $\mathrm{CT}$ and $\mathrm{MRI}$ in recent years, it seems to be commonly accepted that $\mathrm{MRI}$ is better than CT in detecting the skull base, soft tissue and intracranial invasion, ${ }^{2-5} \mathrm{MRI}$ can reflect the regulations of disease progression more accurately, ${ }^{6,7}$ and changes $T$ stage in about $50 \%$ of the patients and clinical stage in $38.6 \%$ of the patients $(70 \%$ of which show upgrade, that means the treatment strategies should be adjusted).,8 Therefore, MRI has become the first choice for $\mathrm{T}$ and $\mathrm{N}$ staging of NPC.

Several studies confirmed that PET-CT should be used as the first choice in detecting distant metastasis. ${ }^{10,11}$ However, the 2008 staging leaves chest radiography, bone scanning and abdominal ultrasound examination as routine imaging

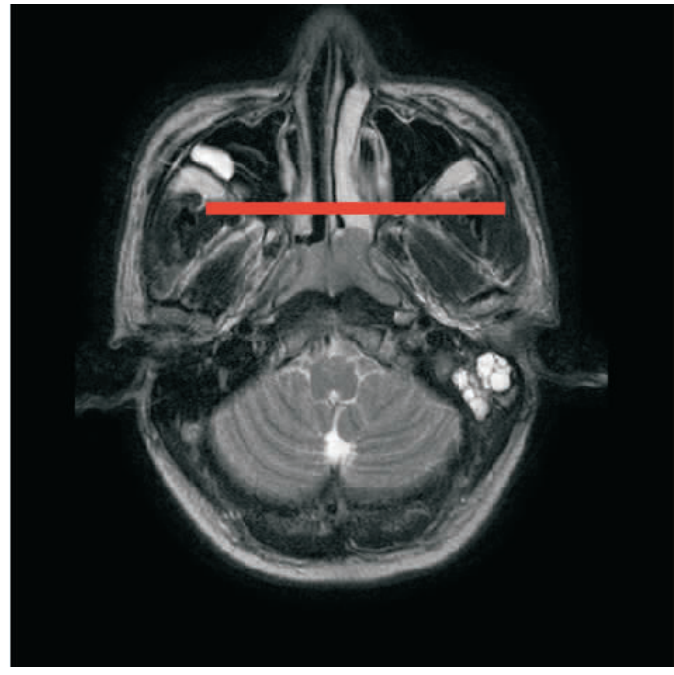

Figure 1 Nasopharyngeal lesions had invaded forward the turbinate, but did not exceed the connection of the bilateral posterior wall of the maxillary sinus

examinations of distant metastasis for $M$ staging due to limited application of PET-CT. It is worth noting that positive PET $-\mathrm{CT}$ results should serve as evidences of M1 although it has not become a routine examination yet.

MRI scanning involves multiple parameters and techniques, and is affected by subjective factors, therefore, appropriate quality control and assurance system must be set up. Hence, the Chinese 2008 staging prescribes detailed scanning specification and report templates while promulgates staging criteria to maximize the diagnostic effectiveness of $\mathrm{MRI}$ and facilitate the exchange of information and summary of data among different units. However, since the staging involves various aspects, the application of these specifications should be further popularized. ${ }^{12}$

Simplification of $\mathrm{T}$ staging. On the basis of the 92 staging, the 2008 staging deletes the prevertebral soft tissue, pterygopalatine fossa, hypopharynx, orbit and other factors, which makes $T$ staging more concise and easy to remember in compliance with the principle of practicability. The deletion is done with the evidences of evidence-based medicine. ${ }^{13}$ Studies have shown that the anterior cervical soft tissue, soft palate, pterygopalatine fossa and orbital invasions are certainly accompanied by other $T$ invasions at the same stage or a higher stage; single pre - or post -cranial group nerve invasions accompanied by other T4 invasions are seen in $85.3 \%$ of NPC patients. It suggests that, deleting factors such as the anterior cervical soft tissue, soft 
palate, pterygopalatine fossa, orbit and no longer distinguishing the pre - or post -cranial nerve invasion, the composition of $\mathrm{T}$ staging would not change and it would not mislead the treatment.

New concepts such as RTOG division of the retropharyngeal lymph nodes and cervical lymph nodes involved in $\mathrm{N}$ staging. A study applying MRI showed that the carotid sheath tumor occupation was constituted by both posterolateral aggression from primary tumor and retropharyngeal lymph node metastasis, ${ }^{14}$ while it is difficult to be distinguished by CT. Therefore, the adscription of retropharyngeal lymph nodes was not involved in the' 92 staging. A series of studies have shown that the occurrence rate of retropharyngeal lymph node metastasis is high, which is the first stop of the metastasis of NPC and is an independent prognostic factor for NO cases, it should be included in N1 sub-phase. 15,16

The application of three -dimensional conformal radiotherapy and intensity -modulated radiotherapy requires radiation doctors to outline lymph node metastasis regions on CT cross -sectional images taking anatomical landmarks into account. The concept coincides with that of surgical division of cervical lymph nodes. The 2008 staging adopted the concept of cervical division, ${ }^{17}$ which makes the staging adapt to and combine with modern treatment modalities with a high practicability.

Problems of the 2008 staging. Although the 2008 staging has made great progresses in staging measures, simplification of $\mathrm{T}$ staging, and new indexes for $\mathrm{N}$ staging, it is believed that there are still some problems in the following aspects.

The definition of the invasion in the nasal cavity and oropharynx should consider the importance of clinical examination. The definition of the invasion in the nasal cavity and oropharynx in the 2008 staging has a bias towards the imaging signs, while misunderstands the anatomic definition. NPC is a malignant tumor occurs in the nasopharyngeal mucosa. It is confined in the mucosa at early stage, and then infiltrates forward into the nasal mucosa and downward into the oropharyngeal mucosa. Clinical and endoscopic examination should be the first choice for detection of mucosal surface lesions.

In the 2008 staging, nasal cavity invasion is defined as the anterior invasion of nasopharyngeal lesion exceeds the connection of bilateral posterior wall of maxillary sinus. Two kinds of nasal cavity invasion are commonly seen: (1) the nodular lesion is enlarged and infiltrates anterosuperior into the nasal septum, middle turbinate and other structures, but may not exceed the connection of bilateral posterior wall of maxillary sinus (Fig. 1); (2) the lesion infiltrates through the lateral wall into the nasal cavity, and is difficult to be detected by MRI. Therefore, we propose to evaluate nasal cavity invasion resort to nasal endoscopy and clinical examination, and imaging definition of nasal cavity invasion should be changed as anterior invasion to nasal structures (turbinate, nasal septum) or exceed the connection of bilateral pterygopalatine fossa. ${ }^{6,8}$

The oropharynx is defined as the intervertebral space between the first and second cervical vertebras in the sixth edition of UICC/AJCC -2002 manual for staging of cancer, ${ }^{18}$ which has been adopted as an international standard. While in the 2008 staging, the oropharynx is defined as the intervertebral space between the second and third cervical vertebras. These inconsistent standards hinder international communication. On the other hand, both standards take imaging bone structures as diagnosis criteria, and the lower edge of the soft palate should be regarded as the boundary between the nasopharynx and oropharynx anatomically. Judging from the site, invasion of nasopharyngeal lesions to the oropharynx usually occurs in the lateral wall. Presentations of cervical vertebras on sagittal and coronal images at the level of oropharyngeal wall are unsatisfactory. The presentations on cross sectional images (subject to the MRI scan angle) need to be referred to. The infiltrating lesions in the oropharyngeal mucosa can be diagnosed through clinical examination.

Definition of "T4 pterygoid muscle and outside masticator space" splits the anatomical definition. In the 2008 staging, the invasion of wing muscles is classified as T3, the invasion of the pterygoid muscle and outside masticator space is classified as T4. Although this classification is more definite than the infratemporal fossa in the ' 92 staging and the infratemporal fossa and masseteric space in the UICC/AJCC staging, it is still not intuitive. Concept of the masticator space is a complete one. If necessary, the split of the concept should be based on evidence-based medicine.Mao et al. found (in " clinical verification of 2008 staging" published in the current issue) that the risk of local recurrence was much higher in T3-wing muscle group than in T3skull base group (lack of intragroup consistency), 
and the risk of recurrence was higher in T3 -wing muscle group than in T4-paranasal sinus and other groups (lack of intergroup risk difference). A staging index should express a structural unit completely and directly, but not to express in a logical type. For example, medial pterygoid muscle as T3, lateral pterygoid muscle as T4. Based on the two points, can we suggest to assign the masticator space in T4 subgroup?

Concepts of cervical division in $\mathrm{N}$ staging are inconsistent with international standards, which impede international communication. Before 1990s, in Head and Neck Surgery, the head and neck lymph nodes were often divided into 12 groups such as submental nodes (including supraclavicular region) according to nerves, blood vessels and other anatomical landmarks. In late 1990s, the American Academy of Otolaryngology-Head and Neck Surgery began to adopt Robbins six-division of the head and neck lymph nodes ( $I-\mathrm{VI}$ regions, without the concept of supraclavicular region). ${ }^{19}$ In 1997, the UICC/AJCC staging of nasopharyngeal carcinoma also identified cervical six-division ( including the concept of supraclavicular region), but the staging was not included in regional standards, the concepts of unilateral/bilateral and supraclavicular regions were still adopted. ${ }^{18}$ In 1999, Som et al. ${ }^{20}$ published cervical lymph node division method based on imaging anatomical marks (including the concept of supraclavicular region). In 1997-2000, Nowak et al. ${ }^{21-}$ 2 and Grègoire et al. ${ }^{23}$ described six-division for preventive irradiation of head and neck tumors (without the concept of supraclavicular region). Several versions of the concept of cervical division have been proposed during the process of evolution from the field of anatomy to surgery, radiology and radiotherapy. To avoid ambiguous definition, authoritative international organizations in Europe and the United States, such as RTOG, jointly issued preventive cervical irradiation field for NO patients with head and neck tumors in 2003 (no concept of supraclavicular region). ${ }^{17}$ Thereafter, multiple studies adopted this standard to investigate the regulation of cervical lymph node metastasis of NPC. ${ }^{24-26}$

The $2008-\mathrm{N}$ staging adopted the division method for preventive cervical irradiation for lymph node-positive patients with head and neck tumor or those underwent operation published by Grègoire et al. in $2006,{ }^{27}$ and made pertinent modifications (without concept of supraclavicular region). However, for the sake of international communication and cooperation, three issues in the $2008 \mathrm{~N}$ staging should be concerned. Firstly, which one we should choose among so many standards of cervical division? We believe that the standard staging issued by authoritative organization (RTOG -2003$)^{17}$ should be selected to make our staging be approved internationally. Secondly, should we copy an intact set of standard or make some practical improvement? To facilitate international communication, we suppose not to change the original criteria if the clinical value of staging would not be affected significantly. Thirdly, how to deal with division standard of supraclavicular region in the UICC/AJCC staging? In the UICC/AJCC staging, supraclavicular region includes the lower half of region IV and region Vb. In 2007, $\mathrm{Ng}$ et al. ${ }^{28}$ have conducted a survival analysis and pointed out that there was a certain practicality of replacing the supraclavicular region with regions IV and Vb. There are inherent risks that the Chinese 2008 staging abandon the concept of supraclavicular region before the UICC/AJCC staging. If UICC/AJCC staging continue to adopt the concept of supraclavicular region, that will give Chinese NPC researchers many inconveniences.

Steps and principles of establishing tumor staging. The establishment of tumor staging includes three steps and four principles: firstly, screening independent prognostic indexes of $\mathrm{T}$ staging and $\mathrm{N}$ staging; secondly, grouping the indexes of $T$ staging and $\mathrm{N}$ staging according to risk of failure follow the principles of case distribution equilibrium, intergroup risk diversity, and intragroup risk consistency; lastly, combining the indexes of $\mathrm{T}$ staging and $\mathrm{N}$ staging follow the principle of predictable prognosis.

Screening indexes of staging should be simple and practical. Indexes of staging should be independent prognostic factors that can reflect the process of tumor progression. The $2008-\mathrm{N}$ staging includes five aspects: the size of retropharyngeal lymph nodes, the size of cervical lymph nodes, extracapsular invasion, unilateral or bilateral lymph node metastasis, involved regions (RTOG six division). However, it is reported that the size of lymph nodes is not an independent prognostic factor for distant metastasis, which only an accompany factor of unilateral or bilateral lymph node metastasis, involved regions and extracapsular invasion. ${ }^{29}$ It should not be included in $\mathrm{N}$ staging to comply with 
the principle of staging simplification. Cranial nerve involvement in $\mathrm{T}$ staging contains MRI diagnosis and clinical diagnosis. ${ }^{30}$

Grouping and combining selected indexes of $\mathrm{T}$ staging and $\mathrm{N}$ staging according to the principles of intergroup risk diversity, intragroup risk consistency and predictable prognosis. When grouping the indexes of $T$ staging, the risk of local recurrence should be mainly considered. The risk of local recurrence could be progressively increased from stage T1 to stage T4 (risk diversity); while subgroups at the same stage should have interlace in survival curves (risk consistency). Based on these principles and survival data, several researchers suggested to downgrade the invasion of oropharynx and nasal cavity to stage $T 1{ }^{31,32}$ In 2009, Mao et al. ${ }^{33}$ re -validated the sixth edition of the UICC/AJCC staging with $\mathrm{MRI}$ data. They verified that the disease-specific survival of T2A and T1 patients are similar, that of T2B and T3 patients are similar, and suggested to combine stages $\mathrm{T} 2 \mathrm{~A}$ and $\mathrm{T} 1$ to $\mathrm{T} 1$, combine stage $\mathrm{T} 2 \mathrm{~B}$ and $\mathrm{T} 3$ to $\mathrm{T} 2$, and downgrade stage $\mathrm{T} 4$ to stage $\mathrm{T} 3$, resulting in three $\mathrm{T}$ stages. The 2008 staging still has four $T$ stages, and the invasion of the nasal cavity and oropharynx remains at stage T2. Although the local recurrence -free survival curves of patients at the four $T$ stages showed no interlace, there was no significant difference among the patients at stages T1, T2, and T3, suggesting there still be room for improvement.

Distant metastasis is the main consideration when grouping the indexes of $\mathrm{N}$ staging. Lee et al. ${ }^{31}$ and Liu et al. ${ }^{32}$ suggested to downgrade the stage $\mathrm{N} 3 a$ in the UICC/AJCC staging to stage N2. This suggestion is similar to the 2008 staging, because the size of lymph nodes can not been included in stage N3. In the 2008 staging, the distant metastasis-free survival curves of patients at four $\mathrm{N}$ stages could be separated well, with significant differences between them.

The final step is staging combination (stage $\mathrm{Tx}$ and stage $\mathrm{Nx}$ are combined to stage $\mathrm{X}$ ). Its principle is to make the overall staging can be used to predict overall survival or disease-specific survival. Via analyzing CT and/or MRI data, Lee et al. ${ }^{31}$ and Liu et al. ${ }^{32}$ proposed to downgrade stage T2NO in sixth edition of the UICC/AJCC staging to stage I, downgrade stage T3NO to stage II, and downgrade stage T4NO to stage III. There are few changes on combination of $\mathrm{T}$ and $\mathrm{N}$ staging and overall staging in the 2008 staging. Differences in disease-specific survival rates between patients at four clinical stages were significant.

Case distribution equilibrium. The equilibrium requires balanced distribution of the patients in four groups: too many patients in one group lead to difficulty in distinguishing the prognosis; too few patients in one group lead to poor practicality. Two papers published by Mao et al. ${ }^{13,29}$ and the paper -clinical validation of the 2008 staging -- published in the current issue are based on data of the same group of patients. Analyzing case distribution, the case distribution of four $\mathrm{T}, \mathrm{N}$, and clinical stages were in equilibrium, with the biggest difference lie in stage N2. The main reason is that stage N2 in the 2008 staging contains indexes of extracapsular invasion and tumor size of $>3 \mathrm{~cm}$, except for unilateral/bilateral lymph node metastasis.

According to literature review and two verification reports in the current issue, comprehensive evaluation of the 2008 staging is as follows. First of all, as a clinical staging standard, the 2008 staging adopts MRI as the main staging measure, includes new independent prognostic factors such as retropharyngeal lymph node metastasis and RTOG cervical division, simplifies $T$ staging, and discards subjective factors. All these changes adapt to modern diagnosis and treatment strategy with high practicality. Secondly, in addition to propose the standards of staging, the 2008 staging also provides scanning standards and report templates for MRI, which helps people understand well and facilitate the popularization of the staging standards. Thirdly, $\mathrm{N}$ staging and overall clinical staging can distinct the risk of distant metastasis and overall survival well. Finally, seeing from the perspective of scientific development, both the 2008 staging and sixth edition of the UICC/AJCC staging are expert consensus built on literature review by the working groups. Study of evidence-based medicine limits to a certain item, without verification of survival data of the overall staging. However, two-step strategy was proposed in the 2008 staging. The second step is to conduct a prospective, multi-center clinical validation on expert consensus and improve to be in line with the development and scientific principle of staging.

The disadvantages of the 2008 staging are that the definition of some anatomical structures, such as the nasal cavity and oropharynx, is limited; the definition of some anatomical structures, such as the 
pterygoid muscle and outside masticator space, is not intuitive enough; some selected indexes lack of evidence of evidence-based medicine; the diversity of local recurrence risk among $T$ stages is unsatisfactory. We should also adopt the international staging when using the 2008 staging for the sake of international communication, while the differences in basic concepts or definitions would trouble the researchers. How to minimize such inconvenience is worth to be thought by Chinese researchers of nasopharyngeal carcinoma staging.

\section{References}

[1] Committee of Chinese Clinical Staging of Nasopharyngeal Carcinoma. Report on the revision of nasopharyngeal carcinoma - 92 staging [J]. Chin J Radiat Oncol, 2009,18(1):2-6. [in Chinese]

[2] Ng SH, Chang TC, Ko SF, et al. Nasopharyngeal carcinoma: MRI and CT assessment $[\mathrm{J}]$. Neuroradiology, 1997,39(10): 741-746.

[3] Poon PY, Tsang VH, Munk PL. Tumour extent and T stage of nasopharyngeal carcinoma: A comparison of magnetic resonance imaging and computed tomographic findings $[\mathrm{J}]$. Head Neck Radiol, 2000, 51(5):287-295.

[4] Sakata K, Hareyama M, Tamakawa M, et al. Prognostic factors of nasopharynx tumors investigated by MR imaging and the value of MR imaging in the newly published TNM staging [J]. Int J Radiat Oncol Biol Phys, 1999, 43(2):273-278.

[5] Sun Y, Ma J, Huang Y, et al. The study of the comparison of CT and MRI in nasopharyngeal carcinoma [J]. Chin J Radiat Oncol, 2005,32(14):788-791. [in Chinese]

[6] King AD, Lam WW, Leung SF, et al. MRI of local disease in nasopharyngeal carcinoma: Tumour extent vs tumour stage $[\mathrm{J}]$. Br J Radiol, 1999, 72 (860): 734-741.

[7] Liang SB, Sun Y, Liu LZ, et al. Extension of local disease in nasopharyngeal carcinoma as detected by magnetic resonance imaging: improvement of clinical target volume delineation $[\mathrm{J}]$. Int J Radiat Oncol Biol Phys, 2009 Feb 27. [Epub ahead of print ], doi : 10.1016/.ijrobp. 2008.11.053)

[8] Liao XB, Mao YP, Liu LZ, et al. How does of Magnetic Resonance Imaging Influence on the 6th Edition of the AJCC Staging System for Nasopharyngeal Carcinoma Compared to Computed Tomography? [J]. Int J Radiat Oncol Biol Phys, $2008,72(5): 1368-1377$.

[9] Sun Y, Mao YP, Ma J, et al. Influences of magnetic resonance imaging on the staging system of nasopharyngeal carcinoma [J]. Aizheng, 2007,26(2):158-163. [in Chinese]

[10] King AD, Ma BB, Yau YY, et al. The impact of F-FDG PET/ CT on assessment of nasopharyngeal carcinoma at diagnosis [J]. Br J Radiol, 2008, 81 (964) : 291-298.

[11] Chang JT, Chan SC, Yen TC, et al. Nasopharyngeal carcinoma staging by ${ }^{[18]} \mathrm{F}$-fluorodeoxyglucose positron emission tomography

[12] [J]. Int J Radiat Oncol Biol Phys, 2005, 62(2):501-507. Dubrulle F, Souillard R, Hermans R. Extension patterns of nasopharyngeal carcinoma $[\mathrm{J}]$. Eur Radiol, 2007, 17 (10): 2622-2630.

[13] Mao YP, Hong MH, Sun Y, et al. Clinical staging of nasopharyngeal carcinoma based on MRI: suggestions [J]. Aizheng, 2007,26(10):1099-1106. [in Chinese]

[14] Liu LZ, Zhang GY, Xie CM, et al. Magnetic Resonance Imaging of Retropharyngeal Lymph Node Metastasis in Nasopharyngeal Carcinoma: Patterns of Spread [J]. Int J Radiat Oncol Biol Phys, 2006, 66(3):721-730.

[15] Ma J, Liu LZ, Tang LL, et al. Retropharyngeal Lymphadenopathy in Nasopharyngeal Carcinoma: Prognostic Value and Staging Categories [J]. Clini Cancer Res, 2007,13 (5) : 1445-1452.

[16] Tang LL, Li L, Mao YP, et al. Retropharyngeal Lymph Node Metastasis in Nasopharyngeal Carcinoma Detected by MRI: Prognostic Value and Staging Categories [J]. Cancer, 2008, $113(2): 347-354$.

[17] Gregoire V, Levendag P, Ang KK, et al. CT-based delineation of lymph node levels and related CTVs in the node-negative neck: DAHANCA, EORTC, GORTEC, NCIC, RTOG consensus guidelines [J]. Radiother Oncol, 2003, 69 (3): 227-236.

[18] Cooper J, Fleming ID, Henson DE, editors. American Joint Committee on Cancer manual for staging of cancer $[M]$. 6th ed. Philadelphia: JB Lippincott. 2002.

[19] Robbins KT, Medina J E, Wolfe GT, et al. Standardizing neck dissection terminology. Official report of the Academy's Committee for Head and Neck Surgery and Oncology [J]. Arch Otolaryngol Head Neck Surg, 1991, 117 (6) :601-605.

[20] Som PM, Curtin HD , Mancuso AA. An imaging based classification for the cervical nodes designed as an adjunct to recent clinically based nodal classifications [J]. Arch Otolaryngol Head Neck Surg, 1999, 125 (4):388-396.

[21] Nowak P, Wijers OB, Lagerwaard FJ, et al. 15. A threedimensional CT-based target definition for elective irradiation of the neck [J]. Int J Radiat Oncol Biol Phys, 1999, 45 (1): 332-339.

[22] Nowak P, Dieren EV, Heijmen B, et al. Treatment portals for elective radiotherapy of the neck: an inventory in the Netherlands [J]. Radiother Oncol, 1997, 43(1):81-86.

[23] Grègoire V, Coche E, Cosnard G, et al. Selection and delineation of lymph node target volumes in head and neck conformal radiotherapy :proposal for standardizing terminology and procedure based on the surgical experience $[\mathrm{J}]$. Radiother Oncol, 2000, 56(2):135-150.

[24] Sun Y, Lu TX, Ma J, et al. The Study on Regulation for the Distribution of Metastatic Cervical Lymph Nodes of Nasopharyngeal Carcinoma $[\mathrm{J}]$. Int J Radiation Oncology Biol Phys, 2005, 63(2 s):363. 
[25] Tang LL, Mao YP, Liu LZ, et al. The volume to be irradiated during selective neck irradiation in nasopharyngeal carcinoma: analysis of the spread patterns in lymph nodes by magnetic resonance imaging $[\mathrm{J}]$. Cancer, 2009, 115(3):680-688.

[26] Wang XS, Li LG, Hu CS, et al. Patterns of level II node metastasis in nasopharyngeal carcinoma $[\mathrm{J}]$. Radiotherapy and Oncology, 2008, 89(1):28-32.

[27] Gregoire V, Eisbruch A, Hamoir M, et al. Proposal for the delineation of the nodal CTV in the node-positive and the postoperative neck [J]. Radiother Oncol, 2006, 79(1):15-20.

[28] Ng WT, Lee AW, Kan WK, et al. N-staging by magnetic resonance imaging for patients with nasopharyngeal carcinoma: pattern of nodal involvement by radiological levels $[\mathrm{J}]$. Radiother Oncol, 2007, 82(1):70-75.

[29] Mao YP, Liang SB, Liu LZ, et al. The N-taging System in Nasopharyngeal Carcinoma with RTOG Guidelines for Lymph Node Levels Based on Magnetic Resonance Imaging [J]. Clini
Cancer Res, 2008, 14(22):7497-7503.

[30] Liu LZ, Liang SB, Li L, et al. Prognostic Impact of Magnetic Resonance Imaging-detected Cranial Nerve Involvement in Nasopharyngeal Carcinoma [J]. Cancer, 2009, $115(9)$ : 19952003.

[31] Lee AW, Au JS, Teo PM, et al. Staging of nasopharyngeal carcinoma: suggestions for improving the current UICC/AJCC Staging System [J]. Clin Oncol, 2004, 16(4):269-276.

[32] Liu MZ, Tang LL, Zong JF, et al. Evaluation of Sixth Edition of AJCC Staging System for Nasopharyngeal Carcinoma and Proposed Improvement $[\mathrm{J}]$. Int J Radiat Oncol Biol Phys, 2008, 70(4): 1115-1123.

[33] Mao YP, Xie FY, Liu LZ, et al. Re-evaluation of the 6th Edition of the AJCC Staging System for Nasopharyngeal Carcinoma and Proposed Improvement based on Magnetic Resonance Imaging [J]. Int J Radiat Oncol Biol Phys, 2009, 73 ( 5 ) : 1326-1334. 